\title{
Author Index Vol. 65, 1995
}

Anderson, J.R. 38 Bechara,G.H. 48 Bloomsmith, M.A. 174 Boehm,C. 43 Braga, J. 144 Butovskaya, M. 30 Cabrera, M.A.A. 54 Corrêa, H.K.M. 25 Coutinho, P.E.G. 25 Cruz,J.B. 48 Ferreira, B.R. 48 Ferreira, F.M. 229 Fontaine,B. 219 Fuentes, A. 172 Goldschmidt, B. 54 Goonan, P.M. 100 Greenfield, L.O. 59 Groves, C.P. 100 Heymann,E.W. 118 Hietanen, J.K. 34 Hooff, J.A.R.A.M. van 121 Hu,H.-g. 138 Jantschke,B. 210 Ji,W.-z. 138 Klaiber-Schuh, A. 210 Knogge,C. 118 Kozintsev, A 30 Lacreuse, A. 202 Lan,H. 138, 154 Lin,S.-y. 138 Linnankoski, I. 34 Liu,A.-h. 138 Liu,R.-q. 138 Llerena, J.C. 54 Ludes, E. 38 Lumsden, W.H.R. 110 Marinho-Filho, J. 115 Moisson,P.Y. 219 Mpongo, E. 43 Müller, K.-H. 224 Muller,M.N. 43 Nunes, A. 85 Phillips, K. A. 159 Pissinatti, A. 48 Pitts, A. 169 Preuschoft, S. 121 Pruetz,J.D. 174

Raps, S. 163 Rodrigues, F.H.G. 115 Shi,L. 154 Sicotte,P. 14 Smith, R.D. 100 Stafford, B.J. 229 Stanford, C.B. 43 Su,B. 138,154 Suomi, S.J. 229 Tenaza,R.R. 172 Terranova, C.J. 181 Wang,W. 138,154 Weinzetl, M. 54 Welker,C. 30,210 Westergaard, G.C. 234 White, F.J. 163 Wickings, E.J. 219 Winkler, L.A. 1 Wright, B.W. 214 Wu,D.-h. 138 Xie,Y.-x. 138 Zhang,W. 154 Zhang,Y.-P. 138 